\title{
Holographic entropy bound from gravitational Fock space truncation
}

\author{
Andreas Aste \\ Department of Physics and Astronomy, University of Basel, 4056 Basel, Switzerland \\ PACS. 11.10.Kk - Field theories in dimensions other than four. \\ PACS. 04.50.th - Gravity in more than four dimensions. \\ PACS. 04.70.-s - Physics of black holes. \\ PACS. 03.67.-a - Quantum information.
}

\begin{abstract}
A simplified derivation of Yurtsever's result, which states that the entropy of a truncated bosonic Fock space is given by a holographic bound when the energy of the Fock states is constrained gravitationally, is given for asymptotically flat spacetimes with arbitrary dimension $d \geq 4$. For this purpose, a scalar field confined to a spherical volume in $d$-dimensional spacetime is considered. Imposing an upper bound on the total energy of the corresponding Fock states which ensures that the system is in a stable configuration against gravitational collapse and imposing a cutoff on the maximum energy of the field modes of the order of the Planck energy leads to an entropy bound of holographic type. A simple derivation of the entropy bound is also given for the fermionic case.
\end{abstract}

The unification of gravity with the other forces in nature remains an elusive goal in quantum field theory. Many recent efforts in this research have been directed at studying theories in which our spacetime is embedded in higher-dimensional manifolds, and it appears that black holes or even more exotic objects like black strings or black branes will play an important role in the understanding of a quantum theory of gravity. An important restriction on possible models of quantum gravity may be supplied by the "holographic principle" $[1,2]$, which asserts basically that the maximum entropy that can be stored inside a bounded region $\mathcal{R}$ in 3 -space is proportional to the surface area $A(\partial \mathcal{R})$ of the region

$$
S_{\max }(\mathcal{R})=\frac{k_{\mathrm{B}}}{4} \frac{A(\partial \mathcal{R})}{l_{p}^{2}},
$$

where $l_{p}=\sqrt{\hbar G / c^{3}}$ is the Planck length and $k_{\mathrm{B}}$ Boltzmann's constant.

The bound is motivated from the observation that the Hawking-Bekenstein entropy of a non-rotating uncharged black hole, which might be considered as the most entropic object within a certain space region, is given by the area $A_{\mathrm{BH}}$ of its event horizon

$$
S_{\mathrm{BH}}=\frac{k_{\mathrm{B}}}{4} \frac{A_{\mathrm{BH}}}{l_{p}^{2}} .
$$


It has been noted that the bound (1) cannot possibly hold for arbitrary spacelike volumes [2-4]. It is possible to construct bounded regions in flat Minkowski space which are contained in curved spacelike hypersurfaces instead of flat spacelike volumes (defined, e.g., by $t=$ const.), such that the area of $\partial \mathcal{R}$ becomes arbitrarily small. A detailed discussion of holographic entropy bounds in a covariant framework has been given in [2]. We will use the simple form (11) of the holographic principle as working hypothesis for special cases in the following.

We consider first a scalar real field $\Phi$ confined to a three-dimensional spacelike cube of size $L$ in Minkowski space, as it has been done previously by Ulvi Yurtsever [5]. The modes of the field are then the solutions of the scalar wave equation $\square \Phi=0$ that vanish on the surface of the cube (alternatively, we could impose periodic boundary conditions). The modes can be labeled by three positive integers $I=\{i, j, k\}$, such that the energy $\epsilon_{I}$ of the corresponding mode is given by

$$
\epsilon_{I}=\hbar \omega_{I}=\frac{\pi \hbar c}{L} \sqrt{i^{2}+j^{2}+k^{2}} .
$$

Summing quantities over the modes can be performed for sufficiently large volumes by the replacement

$$
\sum_{I} \rightarrow \frac{4 \pi}{8} \frac{L^{3}}{\pi^{3} c^{3}} \int d \omega \omega^{2}=\frac{4 \pi V_{c}}{(2 \pi c)^{3}} \int d \omega \omega^{2}
$$

where $V_{c}=L^{3}$ is the volume of the cube.

Going over to a scalar real field confined to a $(d-1)$-dimensional spacelike ball $\mathcal{B}_{d-1}^{\mathrm{R}}$ with radius $R$, which is probably the more natural choice than a cube, where the modes of the field are then the solutions of the free Klein-Gordon equation that vanish on the surface of the ball $\partial \mathcal{B}_{d-1}^{\mathrm{R}}$, i.e. on the sphere $\mathcal{S}_{d-2}^{\mathrm{R}}$, we can generalize the expression (4) for the mode summation to

$$
\sum_{I} \rightarrow \frac{D A_{d-2}(1) V_{d-1}(R)}{(2 \pi c)^{d-1}} \int d \omega \omega^{d-2}
$$

where $V_{d-1}(R)$ is the $(d-1)$-dimensional volume of $\mathcal{B}_{d-1}^{\mathrm{R}}$ and $A_{d-2}(R)$ the $(d-2)$-dimensional volume of the sphere $\mathcal{S}_{d-2}^{\mathrm{R}}$, which can be calculated from

$$
V_{n}(R)=\frac{2 \pi^{n / 2}}{n \Gamma(n / 2)} R^{n}, \quad A_{n-1}(R)=\frac{2 \pi^{n / 2}}{\Gamma(n / 2)} R^{n-1} .
$$

$I$ is now an index labels which the field modes inside the ball. Additionally, we introduced a number $D$ which accounts for further possible polarization or internal symmetry (e.g., color) degrees of freedom.

We will impose now two ad hoc restrictions on the admissible states in the bosonic Fock space that can be constructed from the modes of the scalar field. Firstly, we assume that the maximum energy of admissible modes is of the order of the Planck energy $\epsilon_{p}$, i.e. given by $\mu \epsilon_{p}=\mu \hbar \omega_{p}=\mu\left(\hbar c / l_{p}\right)$, where $\mu$ is of the order of one for naive Planck scale physics. The effective parameter $\mu$ might be much smaller than one, since local quantum field theory could possibly break down at a much larger scale than the Planck length. Secondly, the maximum energy of a state in Fock space should be determined by the energy $E_{\mathrm{BH}}(R)=M_{\mathrm{BH}}(R) c^{2}$ of a black hole with radius $R$. The radius of a non-rotating uncharged black hole in asymptotically flat $d$-dimensional spacetime is related to its mass by [6]

$$
R^{d-3}=\frac{16 \pi G M_{\mathrm{BH}}}{(d-2) A_{d-2}(1) c^{2}},
$$


where $G$ is the gravitational constant (which has a $d$-dependent dimension). Accordingly, we restrict the energy of the Fock states by a maximum energy $E(R)$

$$
E(R)=\frac{\eta(d-2) A_{d-2}(R) c^{4}}{16 \pi G R}
$$

where $\eta$ is of the order of one. In order to calculate the dimension of the truncated Fock space $\mathcal{F}$, we consider first the case where only one specific mode $I$ with single particle energy $\hbar \omega_{I}$ is exited. The maximum number of particles in this mode is then given by the largest integer number which is smaller or equal to $E(R) / \epsilon_{I}$. Therefore, the dimension $\operatorname{dim}\left(\mathcal{F}_{1}\right)$ of the subspace $\mathcal{F}_{1}$ which contains excitations of only one but arbitrary mode is given by

$$
\begin{aligned}
\operatorname{dim}\left(\mathcal{F}_{1}\right) \sim & \sum_{I} \frac{E(R)}{\epsilon_{I}} \sim \frac{\eta D A_{d-2}(1) V_{d-1}(R)}{(2 \pi c)^{d-1}} \int_{0}^{\mu c / l_{p}} d \omega \omega^{d-2} \frac{E(R)}{\hbar \omega}= \\
& \frac{\eta D A_{d-2}^{2}(1) V_{d-1}(1)}{16 \pi(2 \pi)^{d-1}} \mu^{d-2}\left(\frac{R}{l_{p}}\right)^{2 d-4}=: z,
\end{aligned}
$$

where the Planck length is defined in $d$ dimensions by $l_{p}^{d-2}=\hbar G / c^{3}$. As a further step we consider the subspace $\mathcal{F}_{2}$, where two different modes $I$ and $J$ are populated. The corresponding occupation numbers $n_{I}$ and $n_{J}$ must fulfill the condition $n_{I} \epsilon_{I}+n_{J} \epsilon_{J}<E(R)$. It is straightforward to see that there are (up to irrelevant rounding errors) $\frac{1}{2}\left[E(R) / \epsilon_{I}\right]\left[E(R) / \epsilon_{J}\right]$ admissible solutions $\left(n_{I}, n_{J}\right)$ to this condition. Accordingly, the dimension of the subspace $\operatorname{dim}\left(\mathcal{F}_{2}\right)$ which contains excitations of exactly two but arbitrary modes is given by

$$
\operatorname{dim}\left(\mathcal{F}_{2}\right) \sim \frac{1}{2^{2}} \sum_{I} \sum_{J} \frac{E(R)}{\epsilon_{I}} \frac{E(R)}{\epsilon_{J}} \sim \frac{1}{(2 !)^{2}} z^{2} .
$$

The additional prefactor $\frac{1}{2}$ in (10) is necessary to avoid a double counting of the modes. The generalization of the result for $\operatorname{dim}\left(\mathcal{F}_{1}\right)$ and $\operatorname{dim}\left(\mathcal{F}_{2}\right)$ is straightforward. One obtains

$$
\operatorname{dim}\left(\mathcal{F}_{m}\right) \sim \frac{1}{(m !)^{2}} z^{m}
$$

and therefore

$$
W=\operatorname{dim}(\mathcal{F})=\sum_{m=0}^{N} \operatorname{dim}\left(\mathcal{F}_{m}\right) \sim \sum_{m=0}^{\infty} \frac{1}{(m !)^{2}} z^{m}=I_{0}(2 \sqrt{z}),
$$

where $I_{0}$ is the zeroth-order Bessel function of the second kind and $N$ is the number of all modes with energy smaller than $\mu \hbar c / l_{p}$, and for $z \gg 0$ one obtains

$$
W \sim \frac{e^{2 \sqrt{z}}}{\sqrt{4 \pi \sqrt{z}}}
$$

For the bosonic Fock space entropy, we obtain therefore

$$
S_{\mathrm{b}}^{\mathcal{F}}=k_{\mathrm{B}} \log (W) \sim 2 k_{\mathrm{B}} \sqrt{z}
$$


or

$$
S_{\mathrm{b}}^{\mathcal{F}}=k_{\mathrm{B}}\left[\frac{\pi^{(d-3) / 2}}{2^{d-2}(d-1) \Gamma\left(\frac{d-1}{2}\right)^{3}}\right]^{1 / 2} \sqrt{D \mu^{d-2} \eta}\left(\frac{R}{l_{p}}\right)^{d-2} .
$$

For the case $d=4,(15)$ is in agreement with the holographic bound if

$$
D<\frac{3 \pi^{3}}{2} \mu^{2-d} \eta^{-1}
$$

If we assume that $\mu$ and $\eta$ are close to one, then we have $D \leq 46$, compatible with the number of bosonic degrees of freedom that are present in the standard model. The choice $\mu=2 \pi$, the cutoff used in [5], allows for only one bosonic degree of freedom. The result obtained in [5] differs from our result (15) by a geometric factor of the order of one, since a cube was used there as spacelike support of the bosonic field, and an additional factor $(2 \pi)^{(d-2) / 2}$ due to the different energy cutoff. We point out that, e.g., for $d=10$, agreement between the holographic bound, which is assumed to hold for black holes in arbitrary dimensions $d \geq 4$, and (15) is reached for

$$
D<\frac{10418625}{16} \mu^{2-d} \eta^{-1 / 2} \sim 6.5 \cdot 10^{5} \mu^{2-d} \eta^{-1},
$$

but it would be premature to draw any conclusions from this observation. Note also that condition (8) does not exclude energy configurations inside the ball which are gravitationally unstable and which would lead eventually to smaller black holes inside the ball. But it can be shown that such configurations do not contribute to the entropy at the holographic order $\left(R / l_{p}\right)^{d-2}$.

We conclude by giving a simple probabilistic derivation of the entropy bound for a fermionic field confined to a three-dimensional ball with radius $R$, since a derivation is missing in [5] for the fermionic case. The total number of modes below the Planck cutoff is given according to (5) by

$$
N=\frac{4 \pi(4 \pi / 3) R^{3} D}{(2 \pi c)^{3}} \int_{0}^{\mu c / l_{p}} \omega^{2} d \omega=\frac{2 D \mu^{3}}{9 \pi}\left(\frac{R}{l_{p}}\right)^{3},
$$

where $D$ counts again the degeneracy of the modes due to spin degrees of freedom or internal symmetries. We assume now for a while that all these $N$ states are occupied by a fermion. This would lead to a total energy

$$
E_{\text {tot }}=\frac{4 \pi(4 \pi / 3) D R^{3}}{(2 \pi c)^{3}} \int_{0}^{\mu c / l_{p}}(\hbar \omega) \omega^{2} d \omega=\frac{D \mu^{4}}{6 \pi}\left(\frac{R}{l_{p}}\right)^{3} \epsilon_{p},
$$

such that $E_{\text {tot }}$ would be far in excess of the energy bound (8)

$$
E(R)=\frac{\eta}{2}\left(\frac{R}{l_{p}}\right) \epsilon_{p} \quad(d=4) .
$$

Therefore, the occupation density of the admissible modes is extremely sparse. As a further step, we consider a (quasi pure) random process, where we distribute successively more and more particles over the single particle state space. The maximum energy $E(R)$ will be reached for the Fock state generated this way after having added (approximately) $n$ particles, where

$$
n \sim \frac{E(R)}{E_{\mathrm{tot}}} N=\frac{2 \eta}{3 \mu}\left(\frac{R}{l_{p}}\right) .
$$


The entropy of the fermionic Fock space is therefore determined by the number of Fock states with $n$ occupied modes, i.e. by

$$
S_{\mathrm{f}}^{\mathcal{F}} \sim k_{\mathrm{B}} \log (W), \quad W=\left(\begin{array}{c}
N \\
n
\end{array}\right),
$$

since only states with an occupation number very close to $n$ contribute significantly to the total Fock space entropy. From Stirling's factorial approximation formula, one easily derives for binomial coefficients

$$
\left(\begin{array}{l}
N \\
n
\end{array}\right) \sim \frac{e^{n}}{\sqrt{2 \pi n}}\left(\frac{N}{n}\right)^{n} \quad \text { for } N \gg n^{2} \gg 1
$$

and therefore

$$
S_{\mathrm{f}}^{\mathcal{F}} \sim k_{\mathrm{B}} \log \left(\begin{array}{c}
\frac{2 D \mu^{3}}{9 \pi}\left(\frac{R}{l_{p}}\right)^{3} \\
\frac{2 \eta}{3 \mu}\left(\frac{R}{l_{p}}\right)
\end{array}\right) \sim k_{\mathrm{B}} \frac{2 \eta}{3 \mu}\left(\frac{R}{l_{p}}\right)\left[1+\log \left(\frac{D \mu^{4}}{3 \pi \eta} \frac{R^{2}}{l_{p}^{2}}\right)\right] .
$$

Performing the same calculation for a cubic geometry leads to the result given in [5]. For arbitrary spacetime dimensions $d$, one readily derives that the entropy is proportional to $\sim\left(R / l_{p}\right)^{d-3} \log \left(R / l_{p}\right)$.

\section{REFERENCES}

[1] G. 't Hooft, Salamfestschrift 1993:0284-296, gr-qc/9310026.

[2] R. Bousso, Rev. Mod. Phys. 74 (2002) 825-874.

[3] R. Bousso, Class. Quantum Grav. 17 (2000) 997.

[4] E. E. Flanagan, D. Marolf, R. M. Wald, Phys. Rev. D 62 (2000) 084035.

[5] U. Yurtsever, Phys. Rev. Lett. 91 (2003) 041302.

[6] R. C. Myers, M. J. Perry, Annals Phys. 172 (1986) 304. 